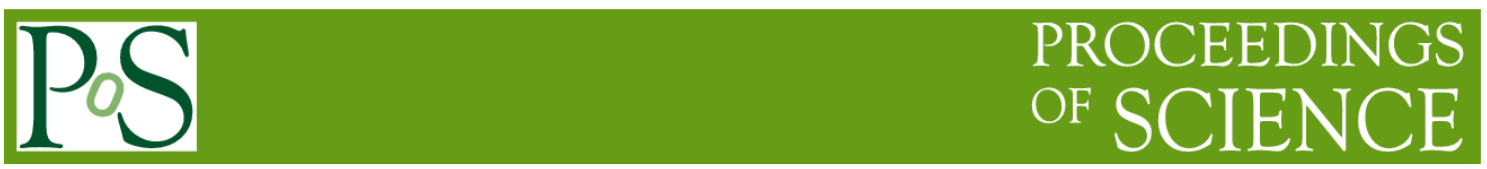

\title{
Latest results on diboson and multiboson production from ATLAS experiment
}

\section{T. A. Kharlamova ${ }^{1}$, on behalf of the ATLAS Collaboration}

Budker Institute of Nuclear Physics

11, Acad. Lavrentieva Pr., Novosibirsk, 630090 Russian Federation

Novosibirsk State University,

2, Pirogova street, Novosibirsk, 630090, Russia

E-mail: tatyana.kharlamova@cern.ch

These proceedings are presenting an overview of the diboson and multiboson production crosssection results obtained by the ATLAS collaboration using proton-proton collisions at the LHC at CERN. The note is focused on the latest measurements of $\mathrm{ZZ}, \mathrm{W}^{ \pm} \mathrm{Z}, \mathrm{W}^{+} \mathrm{W}^{-}$production at $\sqrt{s}=13 \mathrm{TeV}$, of $\mathrm{W}^{+} \mathrm{W}^{-}$and $\mathrm{W}^{ \pm} \mathrm{Z}$ production in the semileptonic final state at $8 \mathrm{TeV}$ and of triboson production at $8 \mathrm{TeV}$. Constraints on anomalous triple and quartic gauge boson couplings obtained from these measurements are briefly discussed as well. At present the measured cross sections are found to be in agreement with the Standard Model predictions within the estimated uncertainties.

Sixth Annual Conference on Large Hadron Collider Physics (LHCP2018)

4-9 June 2018

Bologna, Italy

\footnotetext{
${ }^{1}$ Speaker

(c) Copyright owned by the author(s) under the terms of the Creative Commons 


\section{Introduction}

The measurements of the electroweak gauge bosons production provide a powerful test of the Standard Model (SM) Electroweak (EW) theory at the TeV scale and a precision test of highorder calculations in quantum chromodynamics (QCD). These measurements also help to test directly the gauge boson self-interactions. Any deviation from gauge constraints can cause significant enhancement in the production cross section at high diboson invariant mass due to anomalous triple and quartic gauge boson couplings (aTGC and aQGC). So, the differential cross section measurements allow to perform model-independent new physics search. Also, those processes are backgrounds to other analyses, for example Higgs boson studies, and should be carefully investigated.

The diboson results presented in these proceedings include $\mathrm{ZZ}, \mathrm{W}^{ \pm} \mathrm{Z}, \mathrm{W}^{+} \mathrm{W}^{-}$production at $13 \mathrm{TeV}, \mathrm{W}^{+} \mathrm{W}^{-}$and $\mathrm{W}^{ \pm} \mathrm{Z}$ production in the semileptonic final state at $8 \mathrm{TeV}$ and also triboson production at $8 \mathrm{TeV}$, and are based on the proton-proton collision data collected by the ATLAS detector [1] at the LHC collider [2].

\section{Measurement of $\mathrm{ZZ}$ production at $13 \mathrm{TeV}$}

$\mathrm{Z}$ boson pair production analysis was performed with an integrated luminosity of $36.1 \mathrm{fb}^{-1}$ of data collected in 2015-2016 at center-of-mass energy $\sqrt{s}=13 \mathrm{TeV}$ [3]. Four leptons final states were considered, including $4 \mathrm{e}, 4 \mu, 2 \mathrm{e} 2 \mu$. Both $\mathrm{Z}$ bosons were selected on-shell, with invariant mass of two leptons in the range from 66 to $166 \mathrm{GeV}$. These selected final states have very clean event signature with small background contributions at the level of about $2 \%$. The main background is due to fake leptons and is enhanced in low mass and low $\mathrm{Z}$ boson transverse momentum regions, like most of the signal. Backgrounds were estimated with the Monte Carlo (MC) samples.
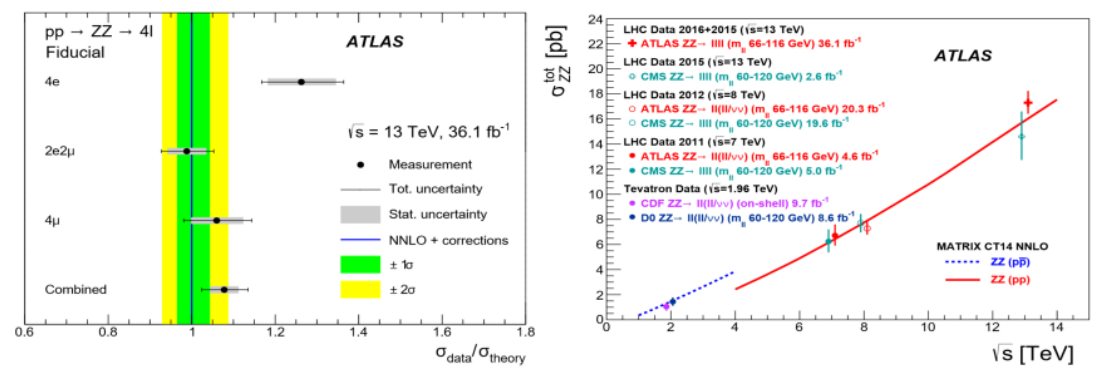

Fig. 1. (Left) Comparison of measured fiducial cross sections to a SM prediction based on an NNLO calculation from the numerical program MATRIX with corrections [3]. (Right) Total cross section compared to other measurements at various center-of-mass energies by ATLAS, CMS, CDF, and D0, and to pure NNLO predictions from the MATRIX program (with no additional higher-order corrections applied) [3]. The total uncertainties of the measurements are shown as bars.

The fiducial cross section $\sigma_{\text {fid }}$ for the reduced phase space was calculated as ratio of signal events found as the difference between numbers of observed events $N_{o b s}$ and estimated backgrounds $N_{b k g}$ to the integrated luminosity $L: \sigma_{f i d}=\frac{N_{o b s}-N_{b k g}}{C \cdot L}$. The factor C is applied to 
correct for detector efficiencies and resolution effects. To calculate cross section a maximumlikelihood fit is used for each channel separately and for all channels combined.

All obtained values are compared with the SM predictions as shown on Fig. 1. The prediction is based on next-to-next-to-leading order (NNLO) calculation provided by MATRIX [4] with gg-initiated contribution correction multiplied by a global next-to-leading order (NLO) correction factor of 1.67, with global NLO EW correction factor of 0.95 (except to the gg-initiated loop-induced contribution) and with contribution of around $2.5 \%$ from the $\mathrm{ZZ}$ production in association with two electroweakly produced jets (EW-ZZjj). As shown on the Fig. 1 (left plot), SM predictions are lower than the data by around $2.5 \sigma$ for $4 \mathrm{e}$ channel and agree with data within $1 \sigma$ for $4 \mu$ and $2 \mathrm{e} 2 \mu$ channels.

The measured fiducial cross section $\sigma_{f i d}$ at $13 \mathrm{TeV}$ was then extrapolated for a total phase space cross section $\sigma_{\text {tot }}$ for $\mathrm{Z}$ bosons with invariant mass from 66 to $166 \mathrm{GeV}$ and all possible SM decay modes: $\sigma_{\text {tot }}=\frac{\sigma_{f i d}}{A_{Z Z} \cdot 4 B r(Z \rightarrow 2 l)^{2}}$. An acceptance correction $A_{Z Z}=0.58 \pm 0.01$ provided by the MATRIX program was applied and the leptonic branching fraction $\operatorname{Br}(Z \rightarrow 2 l)=3.3658 \%$ was taken into account for 4 flavor combinations. The total cross section was $17.3 \pm 0.6$ (stat.) \pm 0.5 (syst.) \pm 0.6 (lumi.) $\mathrm{pb}$, and can be compared with CMS result $17.8 \pm$ 0.6 (stat. $)_{-0.6}^{+0.7}$ (syst.) \pm 0.4 (th. $) \pm 0.5$ (lumi. $) \mathrm{pb}$ [5] and with NNLO with corrections theory prediction $16.9_{-0.5}^{+0.6} \mathrm{pb}$. The comparison is shown on Fig. 1 (right plot), and a good agreement between the data and the SM predictions is observed.

\section{Measurement of $\mathrm{WZ}$ production at $13 \mathrm{TeV}$}

A measurement of $\mathrm{W}^{ \pm} \mathrm{Z}$ production at $13 \mathrm{TeV}$ was performed with an integrated luminosity of $13.3 \mathrm{fb}^{-1}$, with data collected in 2015-2016 at $\sqrt{s}=13 \mathrm{TeV}$ [6]. The analyzed decay channel was $\mathrm{WZ} \rightarrow 3 l$, that includes $3 \mathrm{e}, 3 \mu, \mu 2 \mathrm{e}$, and e $2 \mu$ final states. The $\mathrm{Z}$ boson decaying to two leptons was required to be on-shell. The kinematical quantities of the third lepton, together with the missing transverse energy $\left(\mathrm{E}_{\mathrm{T}}^{\mathrm{miss}}\right)$, were consistent with the $\mathrm{W}$ boson hypothesis. Main backgrounds were of two categories: irreducible backgrounds like $\mathrm{ZZ}, \mathrm{VVV}, \mathrm{t} \overline{\mathrm{t}} \mathrm{t}$, $\mathrm{tZ}$ with three or more prompt leptons were estimated from generated $\mathrm{MC}$ samples and reducible backgrounds, such as $\mathrm{Z}+\mathrm{jets}, \mathrm{Z}+\gamma, \mathrm{W}+\gamma, \mathrm{tt}$, WW with fake or non-prompt lepton, were estimated from data-driven methods. The main systematic uncertainties come out from data-driven background estimation, lepton reconstruction and identification.
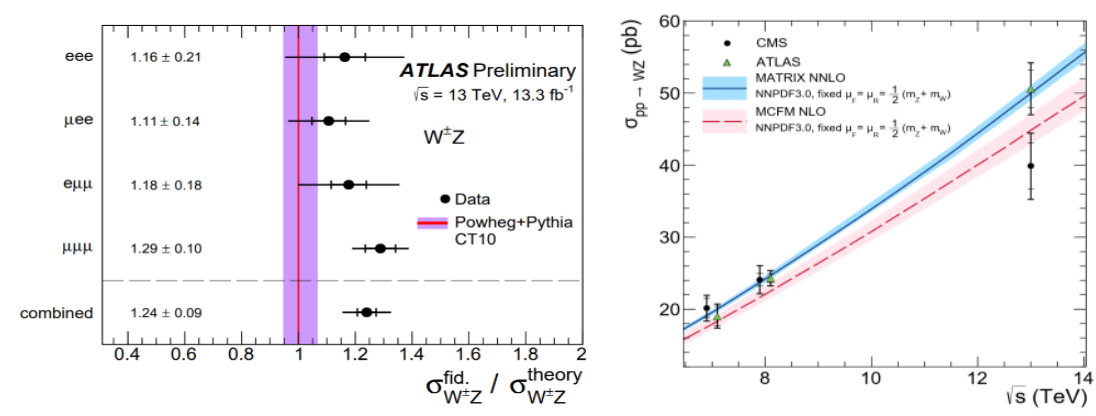

Fig. 2. (Left) Ratio of the measured $\mathrm{W}^{ \pm} \mathrm{Z}$ integrated cross sections in the fiducial phase space to the NLO SM prediction from Powheg+Pythia for each of the four channels and for their combination [7]. (Right) The total pp $\rightarrow \mathrm{WZ}$ cross section as a function of $\sqrt{s}$ measured by the CMS and ATLAS experiments compared to the NLO and NNLO SM predictions [8]. 
On Fig. 2, data to MC fiducial cross sections comparison from [7] is shown and the difference between data and theory prediction has been reduced with new NNLO in QCD calculations by the MATRIX program.

The $\mathrm{W}^{ \pm} \mathrm{Z}$ total cross section was calculated as $50.6 \pm 2.6$ (stat.) \pm 2.0 (syst.) \pm 0.9 (th. $) \pm 1.2$ (lumi.) pb for an integrated luminosity of $3.2 \mathrm{fb}^{-1}$ [6], while the CMS result was $39.9 \pm 3.2$ (stat. $)_{-3.1}^{+2.9}$ (syst.) $\pm 0.4($ th. $) \pm 1.3$ (lumi.) pb for $2.3 \mathrm{fb}^{-1}$ and NNLO calculation made by MATRIX 50.0 $0_{-1.0}^{+1.1}$ (scale) pb [8]. The comparison is shown on Fig. 2 (right). At $13 \mathrm{TeV}$ some tension is seen between ATLAS and CMS results, but those measurements are statistically limited, and more precise comparison require a measurement with increased $13 \mathrm{TeV}$ datasets.

\section{Measurement of $\mathrm{WW}$ production at $13 \mathrm{TeV}$}

Measurement of $\mathrm{W}^{+} \mathrm{W}^{-}$production cross section at $\sqrt{s}=13 \mathrm{TeV}$ was provided with 3.16 $\mathrm{fb}^{-1}$ integrated luminosity of 2015 data in the semileptonic channel $\mathrm{W}^{+} \mathrm{W}^{-} \rightarrow$ $e^{ \pm} v \mu^{\mp} v$ [9]. $E_{T}^{\text {miss }}$ was selected to be greater than $20 \mathrm{GeV}$, and same-flavor leptons $2 \mathrm{e}$ and $2 \mu$ were not considered in the analysis to suppress the Drell-Yan process. No reconstructed jets were required in the event. The main backgrounds were from following sources: the Drell-Yan with fake lepton, $\mathrm{ZZ}$ with missing lepton, $\mathrm{WW} / \mathrm{tt}$ with fake lepton. The signal purity for this process reaches about $74 \%$, and the main background $t \bar{t}$ and single top contribution is about $13 \%$. The main systematic uncertainties are from jet selection and energy scale and resolution.
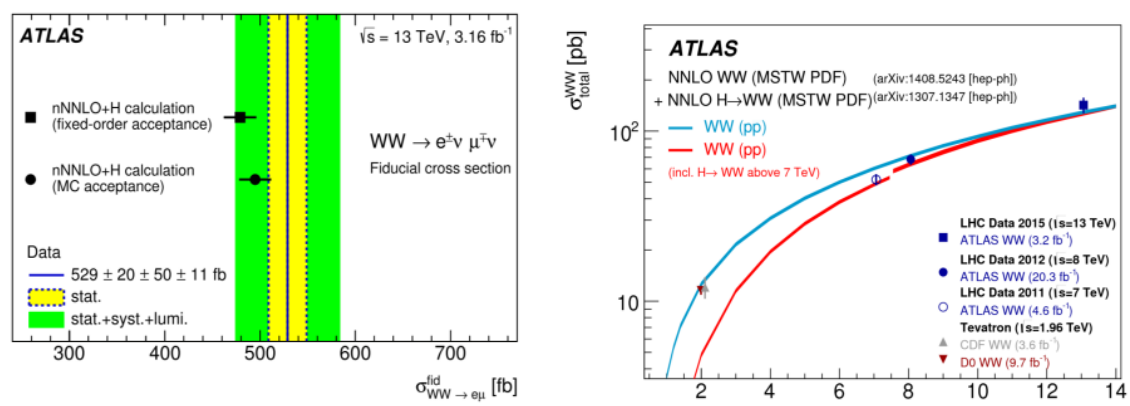

Fig. 3. (Left) The measured fiducial $\mathrm{W}^{+} \mathrm{W}^{-}$cross section at $\sqrt{s}=13 \mathrm{TeV}$ in comparison with the $\mathrm{nNNLO}+\mathrm{H}$ prediction in the fiducial phase space with two different acceptance calculations [9]. (Right) Experimental results for the WW production cross section extrapolated to the full phase space compared to theoretical predictions based on NNLO QCD calculations [9].

On Fig. 3 the fiducial and total $\mathrm{W}^{+} \mathrm{W}^{-}$cross sections are shown. The total cross section at $13 \mathrm{TeV}$ was extrapolated as $142 \pm 5$ (stat.) \pm 13 (syst.) \pm 3 (lumi.) pb [9] and can be compared with the cross section obtained by CMS $115.3 \pm 5.8$ (stat.) \pm 5.7 (syst.) \pm 6.4(th. $) \pm 3.6$ (lumi. ) pb [10]. The theoretical predictions are calculated in the total phase space and include the $\mathrm{q} \overline{\mathrm{q}} \rightarrow \mathrm{WW}$, the non-resonant $\mathrm{gg} \rightarrow \mathrm{WW}$, and the resonant $\mathrm{gg} \rightarrow \mathrm{H} \rightarrow \mathrm{WW}$ subprocesses contributions. The $\mathrm{q} \overline{\mathrm{q}} \rightarrow \mathrm{WW}$ production cross section is known to $\mathrm{O}\left(\alpha_{\mathrm{s}}{ }^{2}\right)$ (NNLO), the non-resonant gg sub-process is known to $\mathrm{O}\left(\alpha_{s}{ }^{3}\right)$, and the resonant $g g \rightarrow \mathrm{H} \rightarrow \mathrm{WW}$ cross section is calculated to $\mathrm{O}\left(\alpha_{\mathrm{s}}{ }^{5}\right)$ taking into account the $\mathrm{H} \rightarrow \mathrm{WW}$ branching fraction. The sum of these sub-processes denoted by nNNLO+H was found to be $128.4_{-3.8}^{+3.5} \mathrm{pb}$, and is in a good agreement with measured cross sections. 


\section{WW and WZ production in the semileptonic final state at $8 \mathrm{TeV}$}

ATLAS have published results on WW and WZ production in the semileptonic final state at $8 \mathrm{TeV}$ [11]. The analysis considers events with exactly one lepton (electron or muon), missing transverse momentum, and either two small-radius jets ( $l v \mathrm{jj}$ decay) or one large-radius jet ( $l v \mathrm{~J}$ decay). The advantage is that the branching ratio for semileptonic decays is about 6 times higher than for fully leptonic ones. This also allows to increase sensitivity to anomalous TGC with high$\mathrm{p}_{\mathrm{T}}$ range available in boosted channel. The dominant background was $\mathrm{W}$ or $\mathrm{Z}$ production with jets and it was quite significant. Both resolved and boosted channels were analyzed independently and then they can be compared with each other and with theory (see Fig. 4).

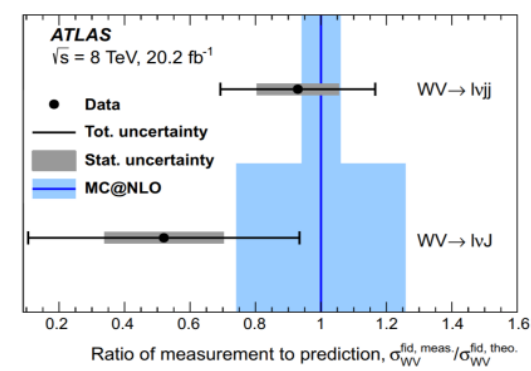

Fig. 4. The ratios of the measured fiducial cross sections to the cross sections predicted by $\mathrm{MC} @ \mathrm{NLO}$, for the WV $\rightarrow 1 v \mathrm{jj}$ and $\mathrm{WV} \rightarrow 1 v \mathrm{~J}$ phase spaces, where $\mathrm{V}$ is $\mathrm{Z}$ or $\mathrm{W}$ [11].

\section{WW $\gamma$ and $\mathrm{WZ} \gamma$ production at $8 \mathrm{TeV}$}

ATLAS published results on $\mathrm{WW} \gamma$ and $\mathrm{WZ} \gamma$ production measurement at $8 \mathrm{TeV}$ [12] for two decay channels: fully leptonic channel $\mathrm{WW} \gamma \rightarrow \mathrm{e} v \mu v \gamma$ with very clean experimental signature and semileptonic channel WW(Z) $\gamma \rightarrow l v \mathrm{jj} \gamma$ with larger branching fraction. The aim was to observe the processes and to search for new physics beyond the SM. For fully leptonic channel the fiducial cross section has been measured to be $\sigma_{\text {fid. }}^{e v \mu v \gamma}=1.5 \pm 0.9$ (stat.) \pm 0.5 (syst.) fb, while the cross sections for semileptonic channel have lower accuracy and so upper limits were derived (see Table 1).

Table 1. Observed and expected cross section upper limits at 95\% CL. The last column shows the theory prediction for the signal cross-section $\left(\sigma_{\text {theo }}\right)$ computed with the VBFNLO [12].

\begin{tabular}{|c|c|c|c|c|}
\hline & & $\begin{array}{l}\text { Observed } \\
\text { limit [fb] }\end{array}$ & $\begin{array}{l}\text { Expected } \\
\text { limit [fb] }\end{array}$ & $\begin{array}{c}\text { SM Prediction } \\
\sigma_{\text {theo }}[\mathrm{fb}]\end{array}$ \\
\hline \multirow[t]{2}{*}{ Fully leptonic } & ev $\mu v \gamma$ & 3.7 & $2.1_{-0.6}^{+0.9}$ & $2.0 \pm 0.1$ \\
\hline & $e v j j \gamma$ & 10 & $16_{-4}^{+6}$ & $2.4 \pm 0.1$ \\
\hline \multirow[t]{2}{*}{ Semileptonic } & $\mu v j j \gamma$ & 8 & $10_{-3}^{+4}$ & $2.2 \pm 0.1$ \\
\hline & evjjy & 6 & $8.4_{-2.4}^{+3.4}$ & $2.3 \pm 0.1$ \\
\hline
\end{tabular}

\section{Anomalous triple and quartic guage couplings measurements}

Anomalous gauge couplings are used in several approaches for different processes. For ZZV (with $\mathrm{V}=\mathrm{Z} / \gamma$ ), couplings are derived from $\mathrm{ZZ}$ and $\mathrm{Z} \gamma$ measurements. The effective vertex function has 4 parameters: two $\mathrm{f}_{4}{ }^{\mathrm{V}}\left(\mathrm{CP}\right.$-violating) and two $\mathrm{f}_{5}{ }^{\mathrm{V}}(\mathrm{CP}$-conserving) for $\mathrm{V}=\mathrm{Z}$ and $\mathrm{V}=$ $\gamma$. Anomalous TGCs increase the $\mathrm{ZZ}$ cross section at high $\mathrm{ZZ}$ invariant masses and high $\mathrm{Z}$ bosons $\mathrm{p}_{\mathrm{T}}$. For WWX process (with $\mathrm{X}=\mathrm{Z} / \gamma$ ) couplings are obtained from $\mathrm{WW}, \mathrm{WZ}$ and $\mathrm{W} \gamma$ measurements. Two approaches are used with 3 parameters for Z. Anomalous TGCs increase the diboson cross section at high $\mathrm{p}_{\mathrm{T}}(\mathrm{V})$ and high $\mathrm{m}_{\mathrm{wv}}$. In the effective theory approach, $\Lambda$ is the scale of new physics; in all the cases, aTGC and aQGC parameters should be zero in SM. Anomalous 
gauge constants can be obtained from the tails of transverse momenta or invariant mass distributions, and the limits on the constants are derived [3, 7, 11].

Quartic couplings such as WVX $\gamma$ (with $\mathrm{V}=\mathrm{Z} / \mathrm{W}, \mathrm{X}=\mathrm{Z} / \gamma$ ) come from $\mathrm{WW} \gamma$ and $\mathrm{WZ} \gamma$ measurements. The effective Lagrangian has 14 parameters. Anomalous QGCs increase WV $\gamma$ production at high $\mathrm{m}_{\mathrm{Wv} \gamma}$ and from those distributions limit on the constants are derived [12].

\section{Conclusions}

Many interesting measurements of dibosons and multibosons production were made by ATLAS at both $8 \mathrm{TeV}$ and $13 \mathrm{TeV}$. Measurements of ZZ, WZ and WW production at $\sqrt{s}=13 \mathrm{TeV}$ are presented along with measurements of $\mathrm{WZ}, \mathrm{Z} \gamma$ and $\mathrm{Z} \gamma \gamma$ production at $\sqrt{s}=8 \mathrm{TeV}$. The measurements are in good agreement with the Standard Model NNLO predictions. Systematic uncertainties are important for precise EW SM measurements. Analysis of new data is providing further improvement of experimental precision and allows more observables to study. Sensitivity for aTGCs and aQGCs is increasing, and no deviation from SM was found yet.

\section{References}

[1] ATLAS Collaboration, The ATLAS Experiment at the CERN Large Hadron Collider, JINST 3 (2008) S08003.

[2] L. Evans and P. Bryant (editors), LHC Machine, JINST 3 (2008) S08001.

[3] ATLAS Collaboration, $Z Z \rightarrow \ell^{+} \ell^{-} \ell^{\prime+} \ell^{\prime-}$ cross-section measurements and search for anomalous triple gauge couplings in 13 TeV pp collisions with the ATLAS detector, Phys. Rev. D 97, 032005

[4] M. Grazzini, S. Kallweit, and D. Rathlev, ZZ production at the LHC: fiducial cross sections and distributions in NNLO QCD, Phys. Lett. B750 (2015) 407 [hep-ph/1507.06257]

[5] CMS Collaboration, Measurements of the $p p \rightarrow Z Z$ production cross section and the $Z \rightarrow 4 \ell$ branching fraction, and constraints on anomalous triple gauge couplings at $\sqrt{s}=13 \mathrm{TeV}$, Eur. Phys. J. C 78 (2018) 165

[6] ATLAS Collaboration, Measurement of the $W^{ \pm} Z$ boson pair-production cross section in pp collisions at $\sqrt{s}=13$ TeV with the ATLAS detector, Phys. Lett. B 762 (2016) 1

[7] ATLAS Collaboration, Measurement of $W^{ \pm} Z$ boson pair-production in pp collisions at $\sqrt{s}=13 \mathrm{TeV}$ with the ATLAS Detector and confidence intervals for anomalous triple gauge boson couplings, ATLAS-CONF-2016-043

[8] CMS Collaboration, Measurement of the WZ production cross section in pp collisions at $\sqrt{s}_{s}=$ $13 \mathrm{TeV}$, Phys. Lett. B 766 (2017) 268 [hep-exp/1607.06943]

[9] ATLAS Collaboration, Measurement of the $W^{+} W^{-}$production cross section in pp collisions at a centre-of-mass energy of $s=13 \mathrm{TeV}$ with the ATLAS experiment, Phys. Lett. B 773 (2017) 35

[10] CMS Collaboration, Measurement of the WW cross section pp collisions at $\sqrt{s}=13 \mathrm{TeV}$, CMS-PASSMP-16-006

[11] ATLAS Collaboration, Measurement of WW/WZ production with the hadronically decaying boson reconstructed as one or two jets in pp collisions at $8 \mathrm{TeV}$ with ATLAS, and constraints on anomalous gauge couplings, Eur. Phys. J. C 77 (2017) 56

[12] ATLAS Collaboration, Study of $W W \gamma$ and WZy production in pp collisions at $\sqrt{s}=8$ TeV and search for anomalous quartic gauge couplings with the ATLAS experiment, Eur. Phys. J. C (2017) 77 